
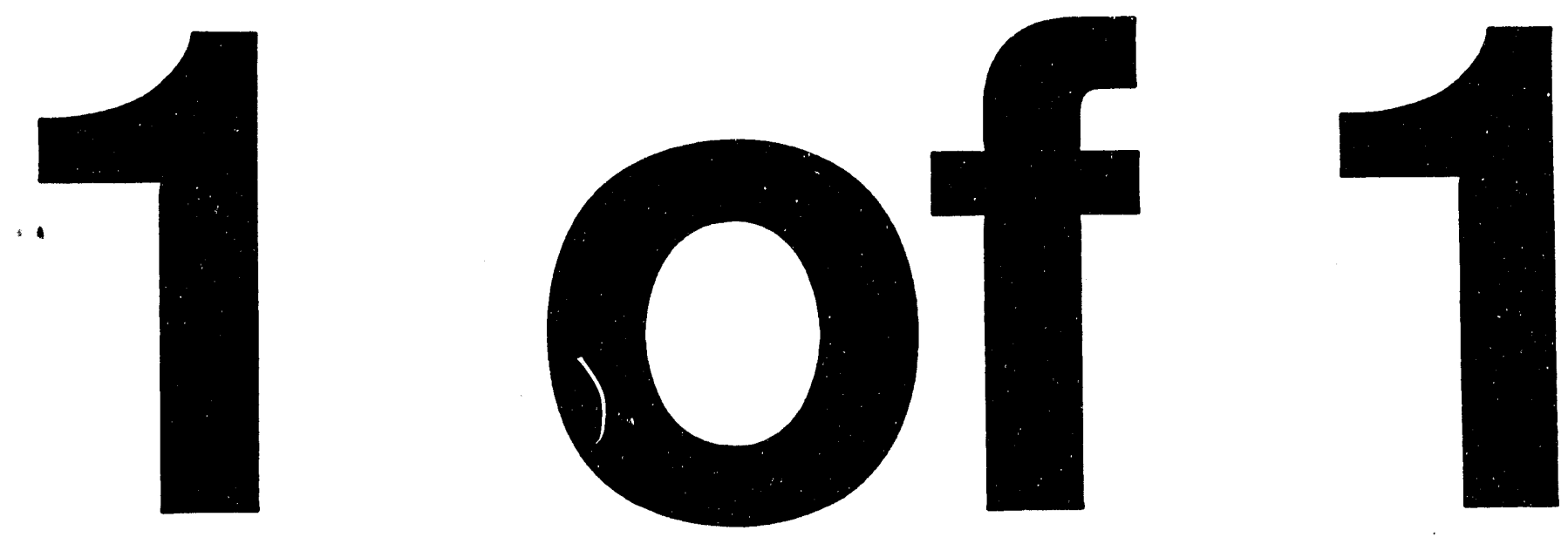
UCRL-JC-117078

PREPRINT

\title{
Computational Model of Drilling with High Radiance Pulsed Lasers
}

\author{
C. D. Boley
}

This paper was prepared for submittal to the International Conference on Applications of Lasers and Electro-Optics

Orlando, Florida

October 17-20, 1994

August 8, 1994

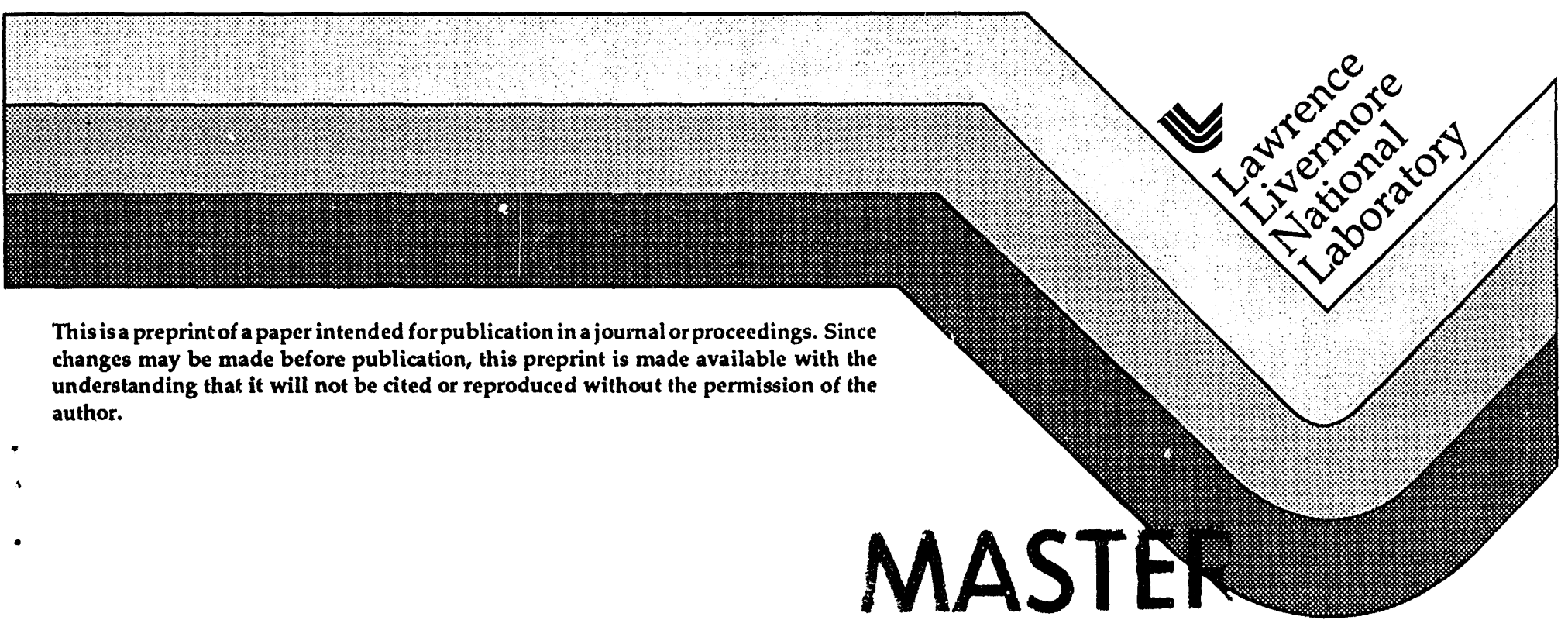

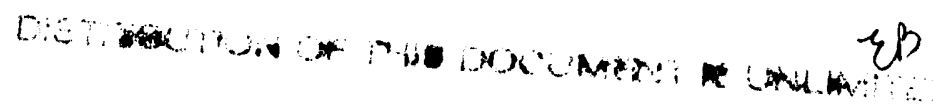




\section{DISCLAIMER}

This document was prepared as an account of work sponsored by an agency if the United States Government. Neither the United States Government nor the University of California nor any of their employees, makes any warranty, express or implied, or assumes any legal liability or responsibility for the accuracy, completeness, or usefulness of any information, apparatus, product, or process disclosed, or represents that its use would not infringe privately owned rights. Reference herein to any specific commercial products, process, or service by trade name, trademark, manufacturer, or otherwise, does not necessarily constitute or imply its endorsement, recommendation, or favoring by the United States Government or the University of California. The views and opinions of authors expressed herein do not necessarily state or reflect those of the United States Government or the University of California, and shall not be used for advertising or product endorsement purposes. 


\title{
Computational Model of Drilling with High Radiance Pulsed Lasers
}

\author{
C. D. Boley \\ University of California \\ Lawrence Livermore National Laboratory \\ Livermore, CA 94550 USA
}

\begin{abstract}
This paper describes a model of drilling by high radiance pulsed lasers. The model contains a one-dimensional description of heat transport below the bottom of the hole, hydrodynamic expansion of the vapor and compressed air, and light propagation through the vapor. The pressure and energy of the vapor are taken from a separate Saha equilibrium code. The boundary conditions at the vaporization surface include the formation of a Knudsen layer within which macroscopic fluid conditions are reached. The absorption mechanisms in the pertinent range of densities and temperatures are photoionization and inverse bremsstrahlung. The model has been applied to the case of drilling in stainless steel with green copper laser light, for peak input intensities ranging from $10^{8}$ to $5 \times 10^{10} \mathrm{~W} / \mathrm{cm}^{2}$. Below $3 \times 10^{8} \mathrm{~W} / \mathrm{cm}^{2}$, there is negligible absorption in the vapor and ablation increases rapidly with intensity. Above this point, ablation still generally increases with intensity, because of a combination of partially penetrating light and electron thermal conduction to the surface. The predicted ablation rates agree semiquantitatively with experiment.
\end{abstract}

\section{Introduction}

In this paper we develop a one-dimensional model of laser drilling with short wavelength, high brightness pulsed lasers. The sides of the hole are envisioned as enforcing 1-D gas dynamics in the expanding vapor. Heat transport below the bottom of the hole is modeled via $1-D$ thermal diffusion.

The absorption of laser energy in the vapor is found to he an important consideration in laser drilling operations. Below a critical incident optical flux, vaporization rates are negligibly influenced by vapor heating. Above this point, laser heating generates a plasma plume which absorbs much of the incident energy. Ablation rates are then determined by the residual light illuminating the surface as well as by the electron thermal conduction to the surface.

A full description of hole drilling would take into account the role of the sides of the hole in absorbing and re-emitting vapor. The present model is, in fact, currently being 


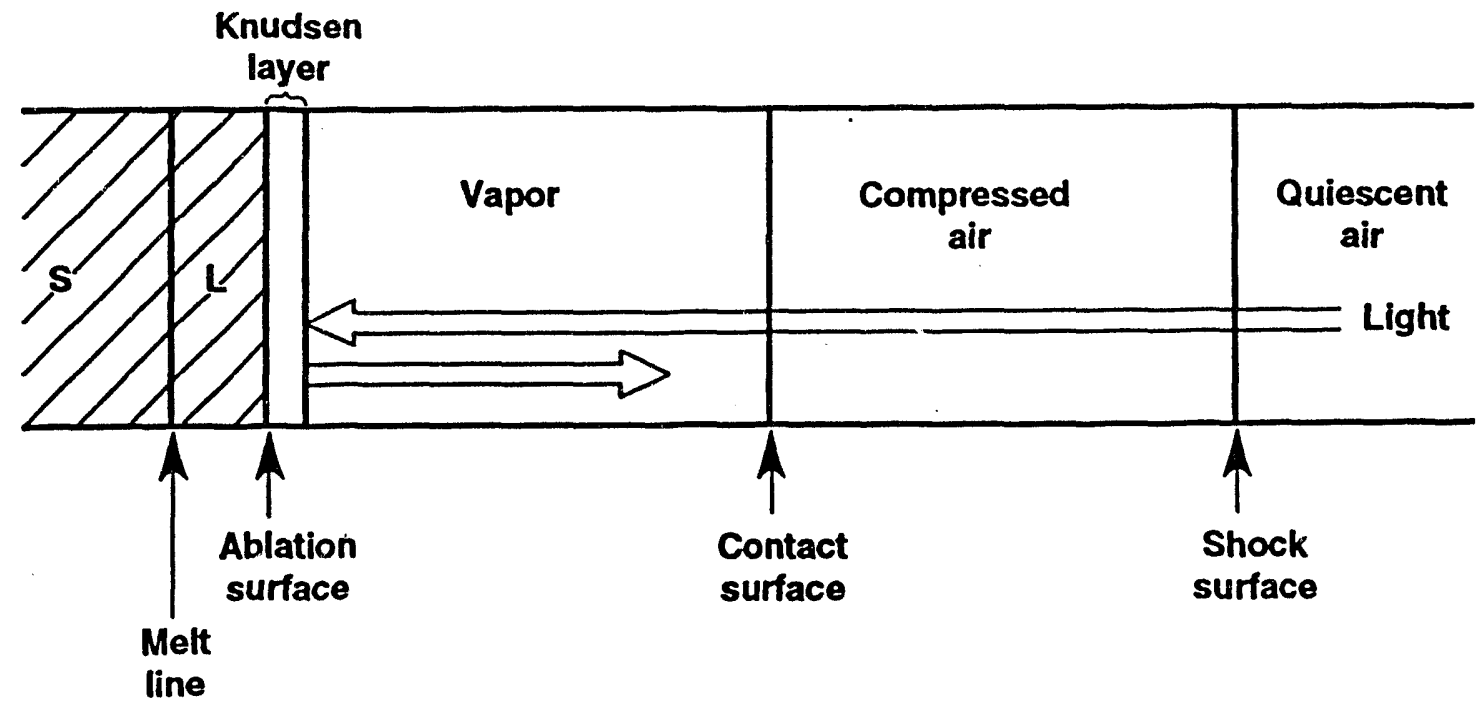

Figure 1: Geometry of hole drilling model (not to scale).

extended in that direction. As it now stands, however, the model is useful for identifying overall trends and for investigeting the role of light-gas and light-surface interactions, the role of boundary conditions, and the sensitivity to materials properties.

The model was suggested by a laser ablation model developed by Kar and Mazumder [1] to study the formation of nanoscale particles. The main differences of the present model are the use of an excitation-ionization equation of state in the vapor, a more detailed treatment of light absorption and of the propagating laser intensity, inclusion of the electron thermal conductivity, and expansion into air rather than into a vacuum. While the gas expansion model of [1] is $2-D$, this is not directly applicable to our problem in the absence of the sides of the hole.

\section{Model}

The geometry of the model is shown in Fig. 1. At the left lies the condensed material. Laser light strikes the surface from the right, causing a melt layer to develop and propagate inward. When the vaporization temperature is reached, the vapor begins to blow off, preceded by a plug of compressed air. This is bounded by a shock front to the far right. When the temperature becomes sufficiently high, both the incident beam and the reflected beam (to the extent that it is appreciable) can be absorbed in the vapor.

In the solid and liquid, the temperature is governed by the 1-D heat conduction equation, with the melt surface moving according to the Stefan condition. In the vapor, we work in terms of the mass density $\rho=m n$, the z-component of the velocity field $u$, and 
the temperature $T$. The hydrodynamic equations are

$$
\begin{gathered}
\frac{\partial \rho}{\partial t}+\frac{\partial}{\partial z}(\rho u)=0 \\
\rho\left(\frac{\partial}{\partial t}+u \frac{\partial}{\partial z}\right) u=-\left(\frac{\partial p}{\partial \rho}\right)_{T} \frac{\partial \rho}{\partial z}-\left(\frac{\partial p}{\partial T}\right)_{\rho} \frac{\partial T}{\partial z} \\
\rho C_{v}\left(\frac{\partial}{\partial t}+u \frac{\partial}{\partial z}\right) T=-T\left(\frac{\partial p}{\partial T}\right)_{\rho} \frac{\partial u}{\partial z}+\frac{\partial}{\partial z} \lambda_{e} \frac{\partial T}{\partial z}+S_{I}-S_{B B}
\end{gathered}
$$

with $C_{v}$ the specific heat per unit mass at constant volume and $S_{I}=\kappa_{\nu}\left[I^{(+)}+I^{(-)}\right]$the bulk heating in the vapor produced by the incident and reflected light (cf. below). Also included is the contribution from the electron thermal conductivity, $\lambda_{e}=5 n_{e} k^{2} T /\left(2 m_{e} n \kappa_{m t}\right)$, with $\kappa_{m t}$ the electron-neutral momentum transfer rate coefficient. Finally, we have included a black-body loss term. In our regime of operations, the vapor is optically thick and this loss is small.

The thermodynamic relations require an equation of state. Since substantial ionization may be present in the bulk of the vapor, the electronic contributions to the pressure and energy density must be considered. Simple estimates show that, for time scales longer than a few nanoseconds, the assumption of local thermodynamic equilibrium is fairly well justified. Hence we have developed and utilized a simple Saha equation of state package, assuming hydrogen-like levels between ionization limits. It agrees with more sophisticated codes in the regime of interest. In Fig. 2(a) we illustrate the predicted electron fraction in iron. Note that at $T=10^{5} \mathrm{C}$ and moderate densities, two or three electrons per atom might be removed. The total pressure is, of course, $p=\left(n+n_{e}\right) k T$.

Similar hydrodynamic equations apply in the plug of compressed air. As the heated vapor pushes on the compressed air, large gradients can develop. We stabilize these by adding an artificial viscosity term [2] to the momentum and energy equations. Since the ionization level of air is low, we handle the air thermodynamics simply via an effective $\gamma \simeq 1.4$, and we neglect laser absorption in the air. At the contact surface between the vapor and the air, the velocity and pressure are continuous. At the shock front, one has the Rankine-Hugoniot relations

$$
\begin{gathered}
v_{a}=\left(c_{a 0} \tilde{p} / \gamma\right)[1+(\gamma+1) \tilde{p} / 2 \gamma]^{-1 / 2}, \\
\rho_{a}=\rho_{a 0}[1+(\gamma+1) \tilde{p} / 2 \gamma][1+(\dot{\gamma}-1) \tilde{p} / 2 \gamma]^{-1},
\end{gathered}
$$

with $\rho_{a 0}$ the density of ambient air, $c_{n 0}$ the speed of sound in ambient air, and $\tilde{p}=p_{a} / p_{0}-1$ the overpressure at the shock. The speed of the shock is related to the overpressure by $\dot{z}_{s}=c_{a 0}[1+(\gamma+1) \tilde{p} / 2 \gamma]^{1 / 2}$.

The incident and reflected light intensities $I^{(+)}$and $I^{(-)}$, respectively, propagate according to

$$
\left(\frac{\partial}{\partial t} \mp c \frac{\partial}{\partial z}\right) I^{( \pm)}=-c \kappa_{\nu} I^{( \pm)}
$$

with $\kappa_{\nu}$ the local absorption coefficient for light of frequency $\nu$. The coordinate system has been set up such that the incident beam propagates in the negative direction. 
Absorption occurs through both photoionization and inverse bremsstrahlung, with the former dominating at lower gas temperatures and the latter taking over after a minimum level of ionization has been established. We employ the treatment of Zel'dovich and Raiser [3], from which it follows that, for an atom with hydrogen-like ionic states, the photoionization absorption coefficient has the form

$$
\kappa_{\nu}^{(P I)}=\frac{2 \alpha^{5}}{3^{3 / 2} \pi} \frac{m_{e} c^{4}}{h \nu^{3}} n S(n, T)[\exp (h \nu / k T)-1][1-\exp (-h \nu / k T)]
$$

where $\alpha \simeq 1 / 137$ is the fine-structure constant and the dimensionless function $S(n, T)$ involves a sum over the ionization states of the atom but is approximately independent of frequency. We tabulate $S(n, T)$ along with the equation of state. Note that for $h \nu \geq k T$, this contribution to the absorption coefficient increases exponentially with frequency. The absorption coefficient for.inverse bremsstrahlung is given by

$$
\kappa_{\nu}^{(I B)}=\frac{\alpha^{3} h^{2} c^{2}}{3 \pi^{2}(6 \pi)^{1 / 2} m_{e}^{3 / 2}} \frac{n_{e}^{2} Z_{e f f}}{(k T)^{1 / 2} \nu^{3}}[1-\exp (-h \nu / k T)],
$$

in terms of the effective charge $Z_{\text {eff }}(n, T)$, which is tabulated along with the equation of state. For $h \nu \geq k T$, this contribution decreases as $\nu^{-3}$. In each of the last two equations, the factor of $[1-\exp (-h \nu / k T)]$ serves to eliminate stimulated emission.

The resulting absorption coefficient is plotted as a function of temperature, for selected deusities, in Fig. 2(b). Note that the contribution from inverse bremsstrahlung becomes increasingly important above about $2 \times 10^{4} \mathrm{C}$.

In modeling the vaporization boundary $z_{v}$, one must combine a microscopic description of the evaporization process with boundary conditions appropriate for fluid flow. Thus we assume that there is a very thin Knudsen layer [4] across which local equilibrium is achieved. We utilize the detailed formulation of Knight [5], in which the boundary conditions on the fluid mass density $\rho_{1}$ and on the fluid temperature $T_{1}$ are found to have the form

$$
\rho_{1}=\rho_{0} f_{\rho}\left(\tilde{u}_{1}\right), \quad T_{1}=T_{0} f_{T}\left(\tilde{u}_{1}\right),
$$

with the 0 subscript referring to the saturated gas, in which $\rho_{0} k T_{0} / m=p_{\text {sat }}\left(T_{0}\right)$. The quantity $\tilde{u}_{1}$ is the boundary value of the velocity, normalized by $\left(2 k T_{1} / m\right)^{1 / 2}$. The functions $f_{\rho}\left(\tilde{u}_{1}\right)$ and $f_{T}\left(\tilde{u}_{1}\right)$, which equal unity for $\tilde{u}_{1}=0$, decrease as the Mach number approaches unity. We assume that vaporization occurs at the local speed of sound, with this speed "turning on" over a small temperature interval beginning at the vaporization point, i.e., $u_{1}=f\left(T_{1} / T_{v}-1\right) c\left(\rho_{1}, T_{1}\right)$, with $f(x)$ an interpolating function which vanishes at the origin and approaches unity as $x$ increases. We also let $f(x)$ approach -1 as $x$ decreases below zero, thus permitting recondensation when the temperature drops below the vaporization point. (In this situation, of course, the Knudsen layer is no longer present.) In practice we choose $f(x)=(2 / \pi) \tan ^{-1}(x / \delta)$, with $\delta=.2$, so that at $20 \%$ above (or below) the vaporization point the fluid is moving at half the speed of sound.

Finally, we employ the hydrodynamic jump conditions across the Knudsen layer. These lead to the "rocket" condition, $\rho_{0} \dot{z}_{v}=-\rho_{1} u_{1}$, and the power condition,

$$
u_{1} \rho_{1}\left(e_{1}+U_{v}+u_{1}^{2} / 2\right)+u_{1} p_{1}=Q
$$



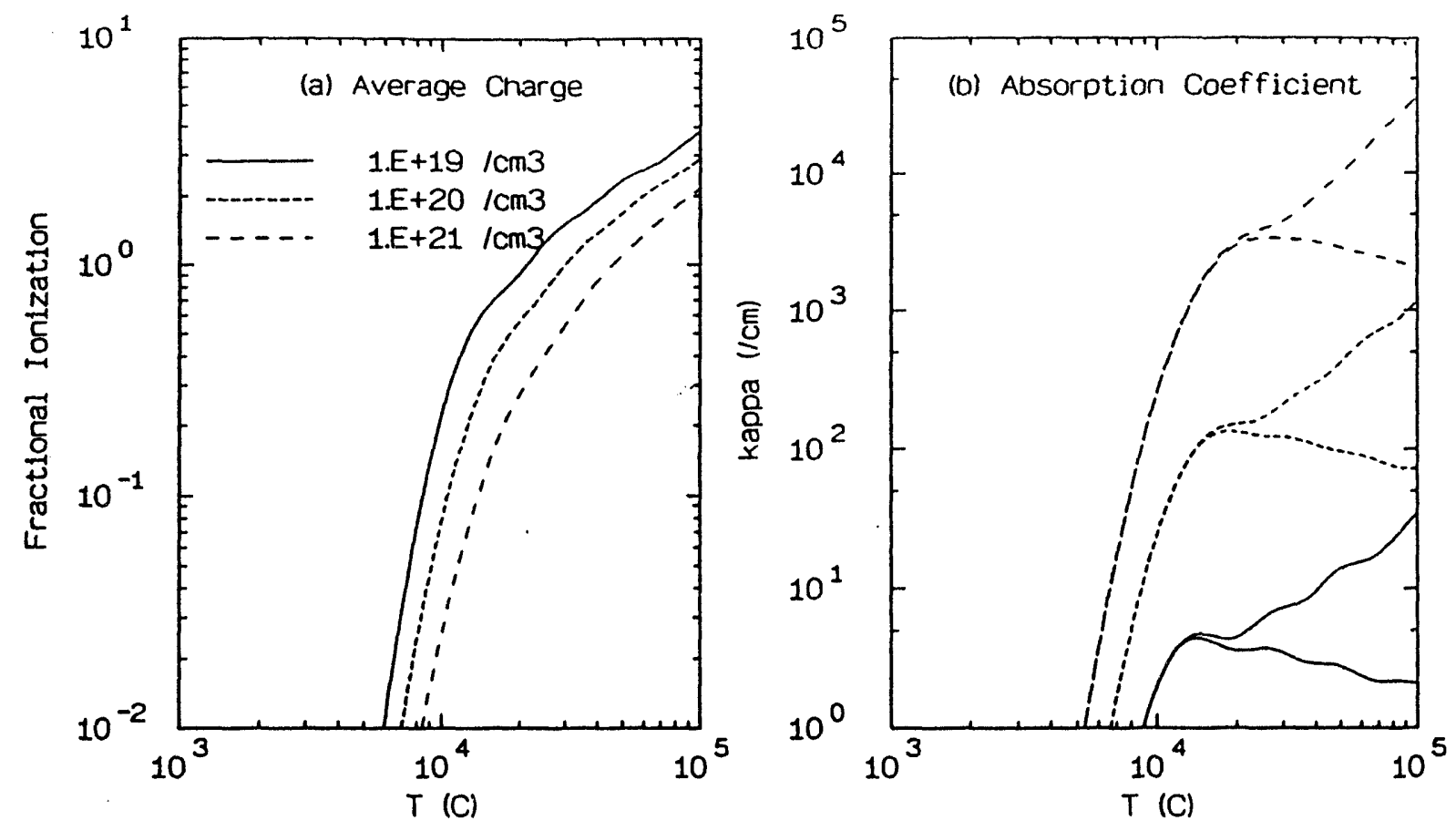

Figure 2: (a) Average charge of vapor, as function of $(n, T)$. (b) Absorption coefficient used in calculations; same densities as in (a). The lower line indicates the contribution from photoionization, while the upper line gives the total.

where $e_{1}=e\left(\rho_{1}, T_{1}\right)$ is the energy per mass in the vapor, $U_{v}$ is the vaporization energy per mass, and $Q$ is the energy flux absorbed at the boundary:

$$
Q=I_{a b s}-\left(\lambda \frac{\partial T}{\partial z}\right)_{0}+\left(\lambda_{e} \frac{\partial T}{\partial z}\right)_{1} .
$$

Here $I_{a b s}$ is the radiation intensity (laser and black body) absorbed at the boundary. We have assumed the gas density to be small compared to the liquid density. (There is also a pressure jump condition, giving the momentum source due to the evaporated material.)

The boundary equations, after discretization, can be solved with a root-finding routine to yield the instantaneous edge temperature. The overall numerical method used in implementing the model is sketched in Appendix A.

\section{Results}

The calculations reported here were performed for a copper laser pulse, of wavelength $510.6 \mathrm{~nm}$, incident on stainless steel. The pulse rises rapidly to a maximum in about $8 \mathrm{~ns}$, falls off almost linearly by $90 \%$ at $70 \mathrm{~ns}$, and has a tail extending to $120 \mathrm{~ns}$ [cf. Fig. 3(c) in the discussion below]. The choices for material data, especially the vapor pressure 
and heat of vaporization, were found to be significant [6]. Our database is discussed in Appendix B. The vapor is modeled as pure iron.

For illustrative purposes, we turn first to the case with a peak power of $I=10^{10} \mathrm{~W} / \mathrm{cm}^{2}$. Figure 3(a) shows the positions of the shock surface and contact surface during the first 2.5 microseconds. During this interval, the average speeds of the two surfaces are about $6 \mathrm{~km} / \mathrm{s}$ and $4 \mathrm{~km} / \mathrm{s}$, respectively. Each surface is beginning to slow down as the vapor and air expand. The typical vapor temperature has decreased from about $10^{5} \mathrm{C}$ at early times to $2 \times 10^{4} \mathrm{C}$ at later times. The air temperature tends to be almost an order of magnitude lower than the vapor temperature. Correspondingly, the flow in the vapor is subsonic, while that in the air is supersonic. During this interval, the pressure at the shock front decreases from 830 to 220 atmospheres. The depths of the melt and vaporization surfaces, relative to the initial surface of the metal, are plotted in Fig. 3(b). The melt layer has reached more than 7 microns and is continuing to expand. The most arresting feature, however, is the fact that, after an initial period of intense ablation, material continues to be slowly ablated even after a few microseconds. The reason for this is revealed by the light absorbed at the surface and the electron thermal conduction to the surface, plotted in Fig. 3(c). While the light is almost entirely blocked after only $8 \mathrm{~ns}$, the thermal flux builds up to a value comparable to the optical flux and is responsible for the subsequent ablation. The black-body flux, by comparison, is four orders of magnitude smaller. The predicted edge temperature, shown in Fig. 3(d), reaches a maximum of $1.2 \times 10^{4} \mathrm{C}$ and is closely synchronized with the absorbed flux.

Making similar runs over a range of input intensities, we obtain the ablation rates illustrated in Fig. 4(a). Intensities beyond $5 \times 10^{10} \mathrm{~W} / \mathrm{cm}^{2}$ were not explored because the resulting vapor temperatures exceeded the limit of our equation of state. Overall, the ablation tends to grow with power, increasing from 0.2 microns at $10^{8} \mathrm{~W} / \mathrm{cm}^{2}$ to 8 microns at the maximum intensity. The model predicts intermediate maxima and minima, to which we now turn. As was pointed out earlier, the peak at low power corresponds to complete transmission of the incident light. The subsequent downward trend results from a shortening of the light pulse to the surface, with thermal conduction here playing a minor role. As the power is raised beyond $6 \times 10^{8} \mathrm{~W} / \mathrm{cm}^{2}$, the vapor expands sufficiently to permit illumination of the surface at late times in the pulse $(t \simeq 60 \mathrm{~ns})$. The decrease in density leads to an enhanced electron thermal conductivity. The resulting electron thermal flux to the surface then becomes comparable to the optical flux. This state of affairs is conveyed in Fig. 4(b), which shows the manner in which both the absorbed optical flux and the thermal flux increase as the power is raised from $6 \times 10^{8}$ to $10^{9} \mathrm{~W} / \mathrm{cm}^{2}$. This trend continues up to $3 \times 10^{9} \mathrm{~W} / \mathrm{cm}^{2}$, beyond which the vapor again becomes opaque after the first several nanoseconds. The subsequent increase in ablation is due to the electron thermal flux.

Experimental ablation rates in stainless steel [7] are shown in Fig. 4(a). These represent single-pulse rates averaged over $10-100$ pulses at about $4 \mathrm{KHz}$, while the calculations pertain to a single pulse starting at an initially ambient temperature. The experimental points tend to increase with power in about the same overall fashion as the predictions, although the evidence is insufficient with regard to the intermediate maxima. In practice, condensation on the walls plays an important role in the drilling of deep holes. 

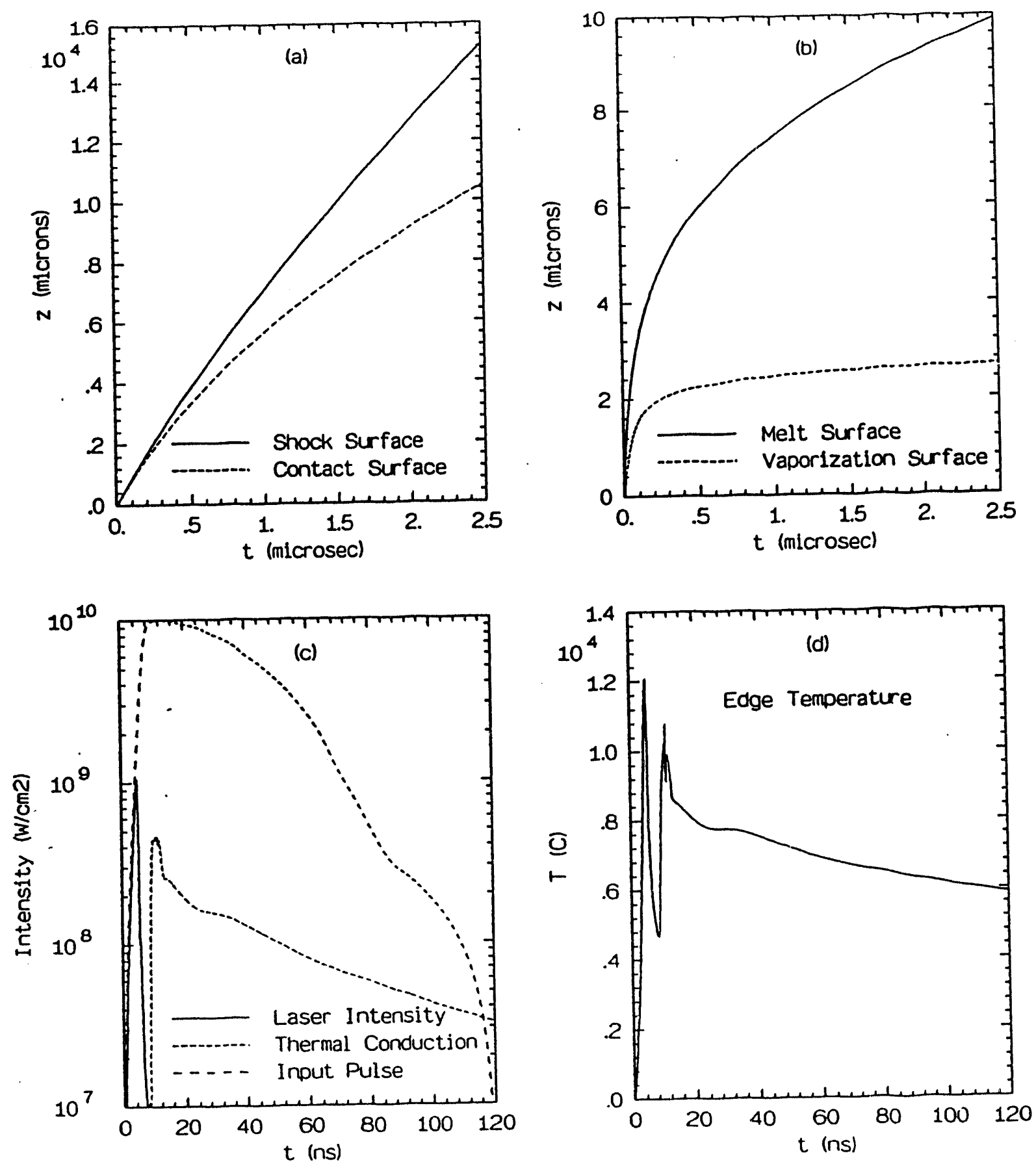

Figure 3: (a) Location of shock surface and contact surface versus time for a peak input power of $10^{10} \mathrm{~W} / \mathrm{cm}^{2}$. (b) Depth of melt surface and vaporization surface. (c) Laser intensity absorbed at surface and electron thermal conduction to surface. Also shown is the input laser intensity. (d) Calculated edge temperature versus time. 

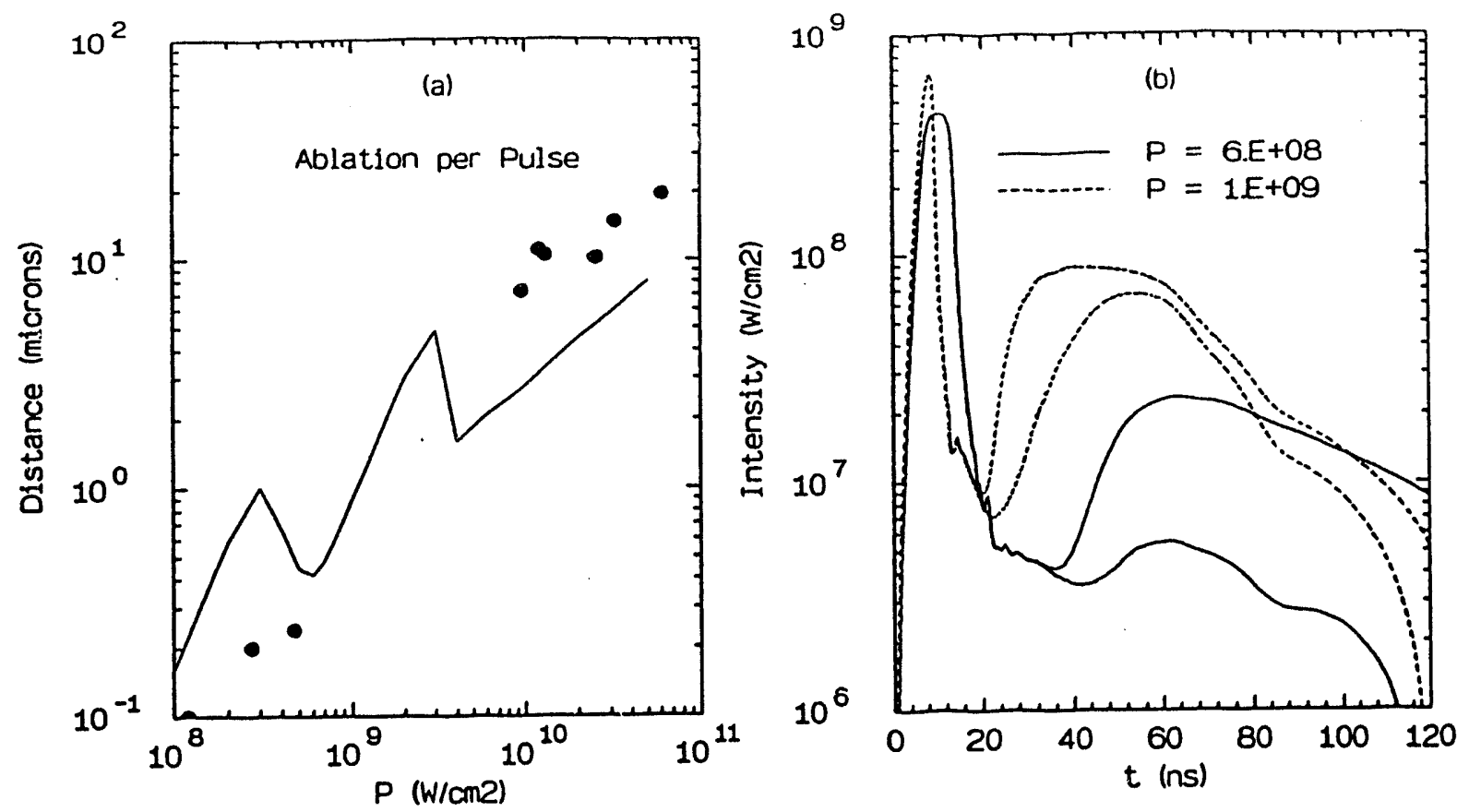

Figure 4: (a) Predicted ablation rate in stainless steel. The circles indicate data of [7]. (b) Optical flux absorbed at surface (lower line) and total absorbed flux (upper line), for two incident laser intensities.

\section{Conclusions}

In this paper, we have presented a one-dimensional model of pulsed laser drilling, including the effects of heat diffusion in the material, hydrodynamic expansion of the vapor and compressed air, and light propagation through the vapor. With green copper laser light illuminating stainless steel, the model predicts minimal absorption in the vapor below an input intensity of $\mathrm{I}=3 \times 10^{8} \mathrm{~W} / \mathrm{cm}^{2}$, and substantial absorption above this point. Up to the highest intensity studied $\left(5 \times 10^{10} \mathrm{~W} / \mathrm{cm}^{2}\right)$, ablation generally increases with power, owing to electron thermal conduction to the surface and penetration of the tail of the laser flux as the vapor expands. Experimental rates appear to exhibit the same overall trend as predicted rates.

\section{Acknowledgements}

The author would like to thank J. J. Chang, J. T. Early, A. C. Hindmarsh, Y. T. Lee, W. A. Molander, R. M. More, and B. E. Warner for useful discussions.

This work was performed under the auspicies of the U.S. Department of Energy by Lawrence Livermore National Laboratory under contract No. W-7405-ENG-48. 


\section{A Numerics}

Our overall numerical approach is to convert the equations of the model into a system of ordinary differential equations in time by suitably differencing the spatial terms, and then to advance the ODEs via a variant of the LSODE solver package [8].

To handle the moving melt, vaporization, contact, and shock surfaces, we introduce dimensionless coordinates [9] in each phase. For example, in the vapor, which lies between $z_{v}$ and $z_{c}$ (the coordinates of the vaporization surface and contact surface, respectively), we take $\xi=\left(z-z_{v}\right) /\left(z_{c}-z_{v}\right)$. When the time and space derivatives are transformed to these coordinates, the moving boundaries are eliminated at the price of adding a nonlinear convective term. We discretize the equations in space with an Eulerian grid attached to $\xi$. The method of differencing the hydrodynamic convective terms requires care. If one attempts central differencing, for example, numerical instabilities develop rapidly. After some experimentation, we employed the pseudocharacteristic method of lines $[10,11]$. In this method, the hydrodynamic equations at each spatial point are written in terms of a $3 \times 3$ matrix multiplying the spatial derivatives. The matrix is readily diagonalized. In the contribution from each eigenvalue, upwind or downwind differencing is then employed, depending on whether the eigenvalue is positive or negative, respectively.

\section{B Material Data}

The available vapor pressure data [12] tend to be limited to temperatures below the boiling point. For higher temperatures, it might at first seem reasonable to employ the form $p_{\text {vap }}(T)=p_{0} \exp (-m U / k T)$, with $U$ equal to the vaporization energy per mass at $T_{v}$ and $p_{0}$ chosen such that the pressure is atmospheric at $T=T_{v}$. Instead, we employ a model [13] in which the vapor pressure has this form but the parameters are chosen so that the curve passes through both the high-temperature data points and the critical point. (For iron, the critical data are $T_{c}=9067 \mathrm{C}$ and $p_{c}=10292 \mathrm{~atm}$.) Near $10000 \mathrm{deg}$, this leads to a vapor pressure exceeding the other estimate by more than an order of magnitude. This form, therefore, leads to a lower predicted edge temperature. In addition, it is argued in [13] that the heat of vaporization should scale with temperature according to $U_{v}(T) / k T_{c}=c\left(1-T / T_{c}\right)^{d}$. The decrease in latent heat with increasing temperature obviously leads to additional ablation.

Thermal conductivity data in materials [14] are generally restricted to temperatures below the melting point. Since the thermal conductivity in a metal beyond its melting point is due primarily to transport by electrons, for which this contribution increases approximately linearly with temperature, we have simply extrapolated in a log-log sense.

The reflectivity is generally unavailable at elevated temperatures, although it is expected to decrease with temperature. It may soon prove feasible to obtain data via femtosecond laser pulses [15]. In the stainless steel runs described here we have employed a reflectivity of .25 . 


\section{References}

[1] A. Kar and J. Mazumder, "A Mathematical Model for Nanoscale Particles Formed During Laser Ablation," Phys. Rev. E 49, 410-419 (1994).

[2] P. J. Roache, "Computational Fluid Dynamics," Hermosa Publishers, Albuquerque, 1972.

[3] Ya. B. Zel'dovich and Yu. P. Raizer, "Physics of Shock Waves and High-Temperature Hydrodynamic Phenomena," Academic Press, 1966.

[4] S. I. Anisimov, "Vaporization of Metal Absorbing Laser Radiation," Sov. Phys. JETP 27, 182-183 (1968).

[5] C. J. Knight, "Theoretical Modeling of Rapid Surface Vaporization with Back Pressure," AIAA J. 17, 519-523 (1979).

[6] The existence and impact of recent expressions for vaporization parameters in metals (Appendix B) were pointed out to the author by J. T. Early.

[7] Data taken at LLNL by L. V. Berzins (low-intensity region) and by E. P. Dragon (high intensities).

[8] A. C. Hindmarsh, "ODEPACK, a Systemized Collection of ODE Solvers," in Scientific Computing, R. S. Stepleman et al. (eds.), North-Holland, Amsterdam, 1983 (Vol. I of IMACS Transactions on Scientific Computing), pp. 55-64.

[9] H. G. Landau, "Heat Conduction in a Melting Solid," Quart. Appl. Math. 8, 81 (1950).

[10] M. B. Carver, "Pseudo Characteristic Method of Lines Solution of the Conservation Equations," J. Comp. Phys. 35, 57-76 (1980).

[11] Y.-Z. Wang and S. H. Johnson, "Pseudocharacteristic Method of Lines Simulation of Single- and Two-Phase One-Dimensional Flow Transients," Proc. IMACS 11 th World Congress, Vol. 2, pp 201-204, Oslo, 1985.

[12] A. Nesmeyanov, "Vapor Pressure of the Chemical Elements," Elsevier Publishing Co., 1963.

[13] K. Hornung, "Liquid Metal Coexistence Properties from Corresponding States and Third Law," J. Appl. Phys. 46, 2548 (1975).

[14] Y. S. Touloukian et al., "Thermophysical Properties of Matter, Vol. I. Thermal Conductivity - Metallic Elements and Alloys," IFI/Plenum, 1970.

[15] A. Ng. et al., "Reflectivity of Intense Femtosecond Laser Pulses from a Simple Metal," Phys. Rev. Letters 72, 3351 (1994). 

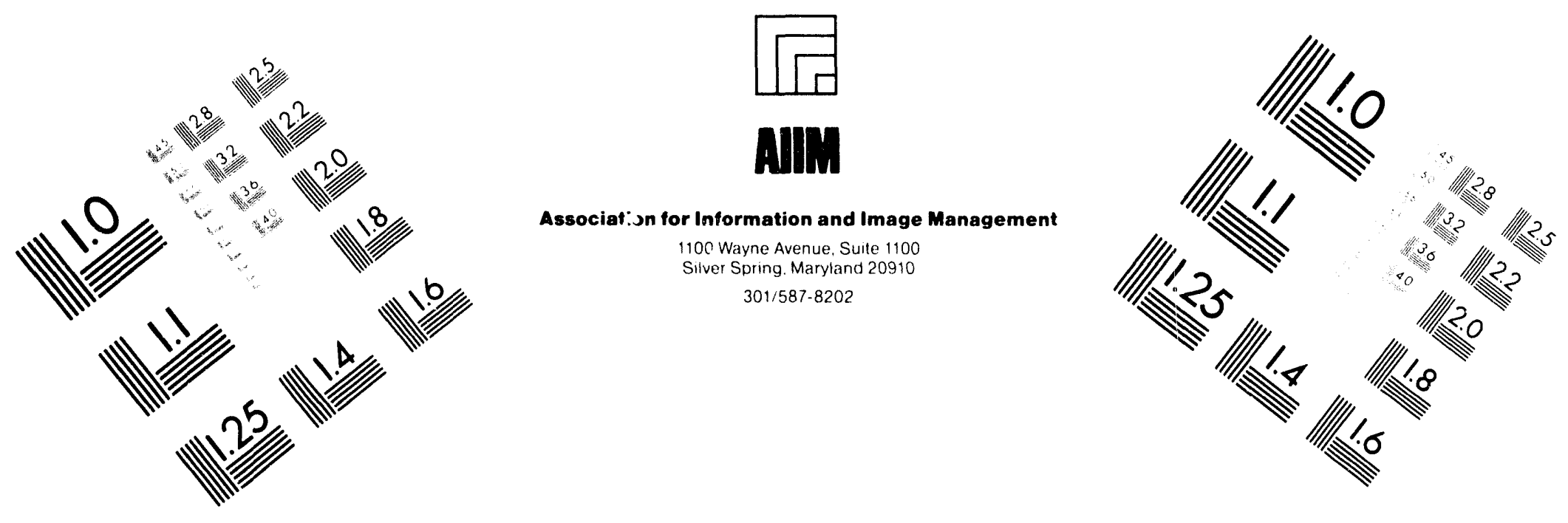

\section{Centimeter}

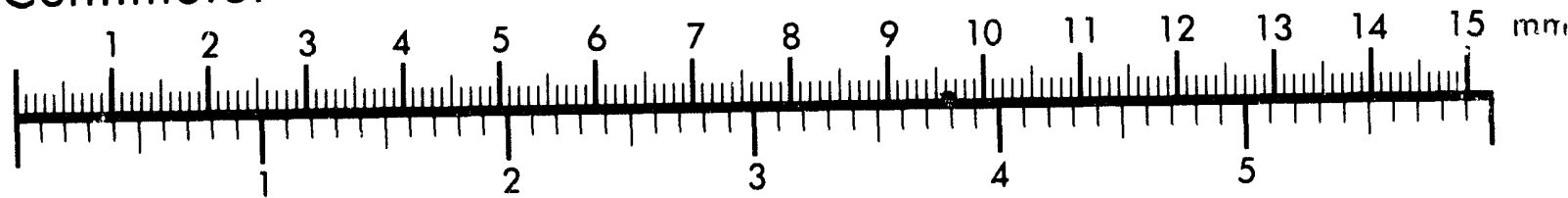

Inches
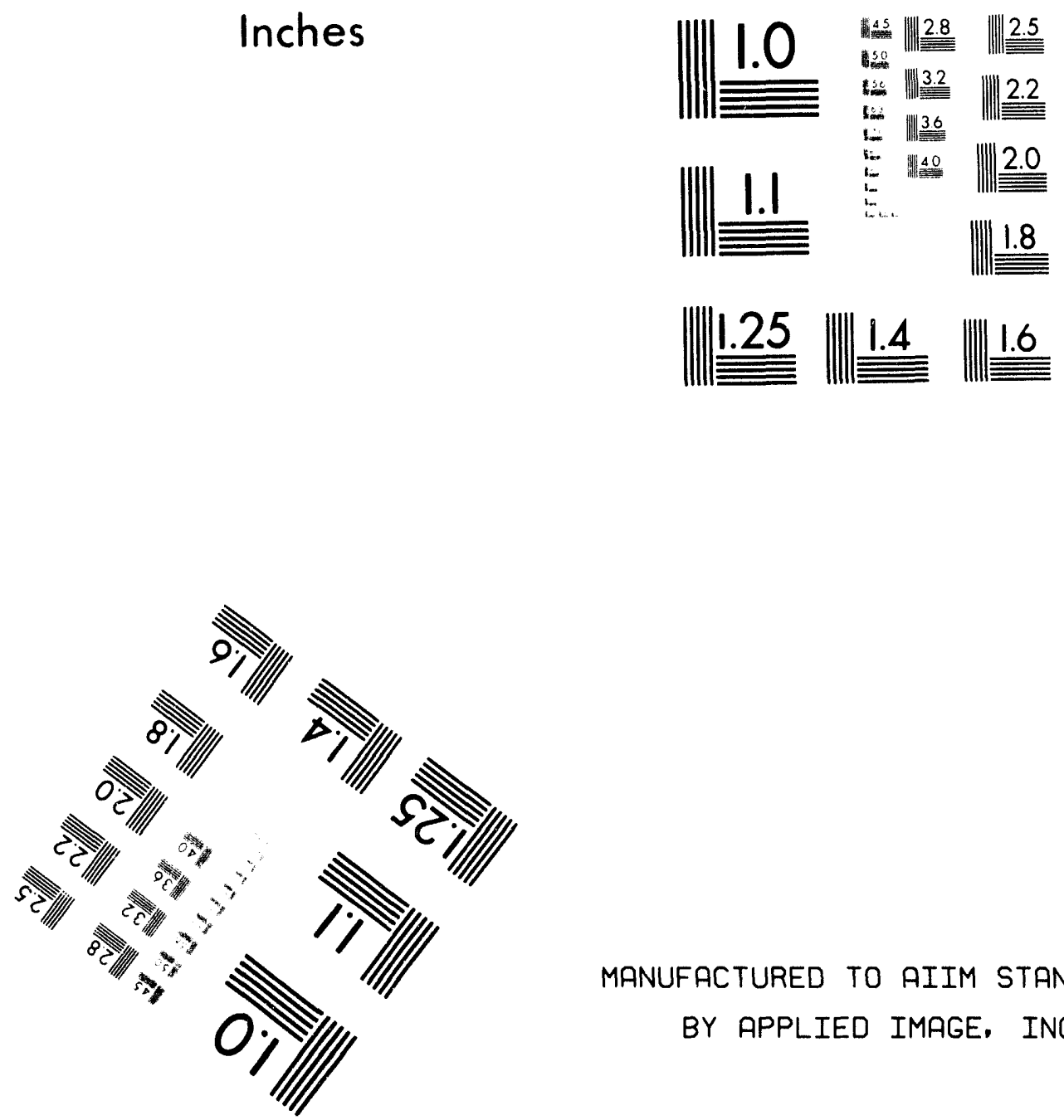

MANUFACTURED TO AIIM STANDARDS

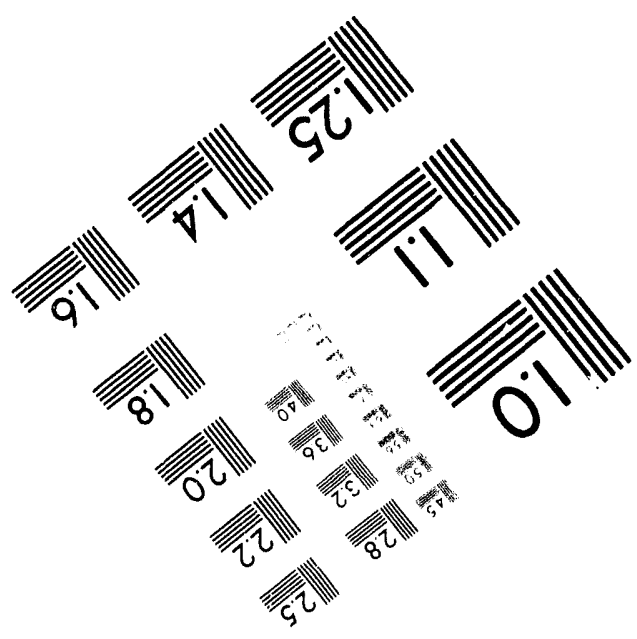




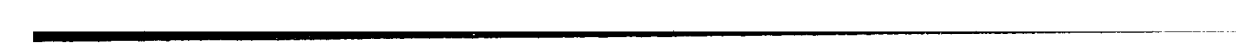



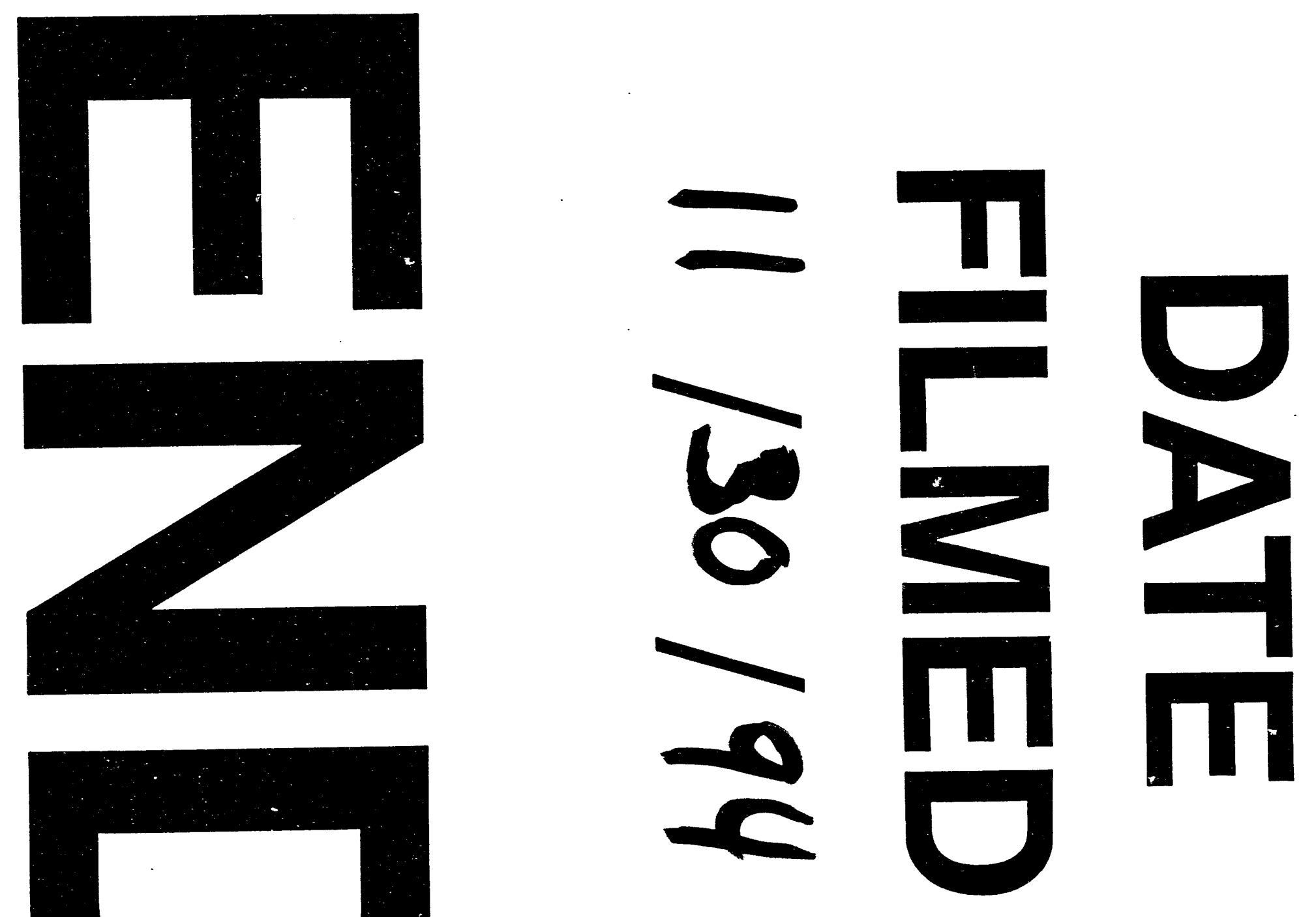